\title{
ON THE SELF-INTERSECTIONS OF THE IMAGE OF THE UNIT CIRCLE UNDER
}

\section{A POLYNOMIAL MAPPING}

\author{
J. R. QUINE ${ }^{1}$
}

ABSTRACT. We prove that if $p$ is a polynomial of degree $n$, then with certain exceptions the image of the unit circle under the mapping $p$ has at most $(n-1)^{2}$ points of self-intersection. We apply our method to the problem of computing polynomials univalent in $|z|<1$.

Introduction. Let $p(z)$ be a polynomial in the complex variable $z$.

Definition. We say $w$ is a vertex, or point of self-intersection of the curve $p\left(e^{i \phi}\right), 0 \leqq \phi \leqq 2 \pi$, if there exist $z_{1}$ and $z_{2}$ distinct with $\left|z_{1}\right|=\left|z_{2}\right|=1$ such that $p\left(z_{1}\right)=p\left(z_{2}\right)=w$.

C. J. Titus [6, p. 60] conjectured that if the degree of $p$ is $n$, then with certain exceptions the curve $p\left(e^{i \phi}\right)$ has at most $(n-1)^{2}$ vertices. Our main result is a proof of this conjecture. We also show how the methods used relate to the problem of finding polynomials univalent in $|z|<1$.

Computation of vertices. Let $p(z)=a_{0}+a_{1} z+\cdots+a_{n} z^{n}$. In order to study the vertices of $p\left(e^{i \phi}\right)$, we study the polynomial

$$
G\left(z_{1}, z_{2}\right)=\left(p\left(z_{2}\right)-p\left(z_{1}\right)\right) /\left(z_{2}-z_{1}\right) .
$$

We find it convenient following Dieudonné [3] to make a transformation of the variables $z_{1}$ and $z_{2}$. Let

$$
\begin{aligned}
& A=\{(t, x)|-1<t<1,| x \mid=1\}, \\
& B=\left\{\left(z_{1}, z_{2}\right)|| z_{1}|=| z_{2} \mid=1, z_{1} \neq z_{2}\right\} .
\end{aligned}
$$

Let $\psi: A \rightarrow B$ send the pair $(t, x)$ onto the pair $\left(x e^{i \theta}, x e^{-i \theta}\right)$ where $\theta=$ arc $\cos t$, i.e. $t=\cos \theta$ and $0 \leqq \theta \leqq \pi$. Geometrically $x$ is the midpoint of the

Presented to the Society, March 31, 1972 under the title On the double points of the image of the unit circle under a polynomial mapping; received by the editors April 3, 1972 and, in revised form, July 2, 1972.

AMS (MOS) subject classifications (1970). Primary 30A90, 30A06, 30A34, 30A32; Secondary 57D40.

Key words and phrases. Coefficient problem, univalent polynomials, points of selfintersection.

${ }^{1}$ This paper is part of the author's doctoral dissertation at the University of Michigan written under Professor F. W. Gehring.

(c) American Mathematical Society 1973 
arc of the unit circle from $z_{1}$ to $z_{2}$ in a counterclockwise direction. The number $\theta$ is the length of arc along the unit circle from $x$ to $z_{1}$ or $z_{2}$. Thus $\psi$ is one-to-one and onto. If $\psi:(t, x) \rightarrow\left(z_{1}, z_{2}\right)$ and $\theta=\arccos t$, we have

$$
\begin{aligned}
G\left(z_{1}, z_{2}\right) & =\left(p\left(x e^{i \theta}\right)-p\left(x e^{-i \theta}\right)\right) / x\left(e^{i \theta}-e^{-i \theta}\right) \\
& =\sum_{k=0}^{n-1} a_{k+1} \frac{\sin (k+1) \theta}{\sin \theta} x^{k}=\sum_{k=0}^{n-1} a_{k+1} U_{k}(t) x^{k}
\end{aligned}
$$

where $U_{k}(t)=\sin (k+1) \theta / \sin \theta$ is the $k$ th Chebyshev polynomial of the second kind. We define the Dieudonné polynomial associated with $p$ to be the polynomial $g(t, x)=\sum_{k=0}^{n-1} b_{k}(t) x^{k}$ where $b_{k}(t)=a_{k+1} U_{k}(t)$. We note that $g(1, x)=p^{\prime}(x)$. For $\left(z_{1}, z_{2}\right) \in B$ and $\psi:(t, x) \rightarrow\left(z_{1}, z_{2}\right)$, we see that

$$
G\left(z_{1}, z_{2}\right)=G\left(z_{2}, z_{1}\right)=g(t, x)=g(-t,-x) .
$$

If $w=p\left(z_{1}\right)=p\left(z_{2}\right)$ is a vertex of $p\left(e^{i \theta}\right)$ then all of the above expressions are zero and we say that $w$ corresponds to the two pairs $\left(z_{1}, z_{2}\right)$ and $\left(z_{2}, z_{1}\right)$ in $B$ or the two pairs $(t, x)$ and $(-t,-x)$ in $A$. A vertex may correspond to more than two of these pairs, for example if $w=p\left(z_{1}\right)=p\left(z_{2}\right)=p\left(z_{3}\right)$ for $\left|z_{1}\right|=\left|z_{2}\right|=\left|z_{3}\right|=1$ and $z_{1}, z_{2}$ and $z_{3}$ distinct; however $N$ distinct vertices must correspond to at least $2 N$ distinct pairs.

To compute the vertices of $p\left(e^{i \phi}\right)$, we must find pairs $(t, x)$ in $A$, such that $g(t, x)=0$. We define

$$
g^{*}(t, x)=x^{n-1} \overline{g(\tilde{t}, 1 / \bar{x})}=\sum_{k=0}^{n-1} \bar{a}_{n-k} U_{n-k-1}(t) x^{k} .
$$

If $t$ is real and $x_{1}, x_{2}, \cdots, x_{n-1}$ are the zeros of $g(t, x)$, then $1 / \bar{x}_{1}, \cdots$, $1 / \bar{x}_{n-1}$ are the zeros of $g^{*}(t, x)$, thus if $t_{0}$ is a fixed real number, then $g\left(t_{0}, x\right)$ has a zero $x_{0}$ on $|x|=1$ only if $g\left(t_{0}, x\right)$ and $g^{*}\left(t_{0}, x\right)$ have the common zero $x_{0}$. This can happen only if $R\left(t_{0}\right)=0$ where $R(t)$ is the resultant of $g(t, x)$ and $g^{*}(t, x)$ as polynomials in $x$. (See B. L. van der Waerden [8, p. 84].) Thus if $(t, x) \in A$ and $g(t, x)=0$ then $R(t)=0$. We are therefore interested in the roots of $R(t)$ in the interval $-1<t<1$. If we look at the $2(n-1) \times 2(n-1)$ determinant expression for $R(t)$ we note that the product of the entries on the major diagonal is $\left|b_{n-1}(t)\right|^{2(n-1)}$. Since $b_{n-1}(t)=a_{n} U_{n-1}(t)=a_{n}\left(2^{n-1} t^{n-1}+\cdots\right)$, we have that the degree of $R(t)$ is $2(n-1)^{2}$ if $a_{n} \neq 0$. Now every vertex $p\left(e^{i \phi}\right)$ corresponds to at least two zeros $(t, x)$ and $(-t,-x)$ in $A$ of $g(t, x)$ and therefore to at least two zeros, $t$ and $-t$, of $R(t)$. Suppose $p\left(e^{i \phi}\right)$ has $N$ vertices. If we could conclude that the $2 N$ roots of $R$ corresponding to these vertices were distinct then we would have $N \leqq(n-1)^{2}$. If the roots were not distinct it seems likely that we could vary the polynomial slightly to make the roots distinct without decreasing the number of vertices. This establishes the likelihood of the 
upper bound $(n-1)^{2}$. In the proof given below we use a simple version of Bézout's theorem, a classical theorem in the theory of algebraic curves, which effectively sidesteps the difficulties of an argument based on the above outline. The proof of Bézout's theorem, in fact, uses the resultant in much the same way as we outlined above (Walker [9, p. 59]).

Main theorem. We now prove that with certain exceptions $(n-1)^{2}$ is the desired upper bound. That there are exceptions can be seen by taking $p(z)=z^{2}$. In this case $p(z)=p(-z)$ for all $z$, and every point of the curve $p\left(e^{i \phi}\right)$ is a vertex. In fact if $p(z)=g\left(z^{m}\right)$ for some polynomial $g$ and some integer $m>1$, then $p(z) \equiv p\left(z e^{2 \pi i / m}\right)$ and again every point of the curve $p\left(e^{i \phi}\right)$ is a vertex.

THEOREM 1. If $p(z)$ is a polynomial of degree $n$ and not of the form $g\left(z^{m}\right)$ where $g$ is a polynomial and $m$ is an integer, $m>1$, then the curve $p\left(e^{i \phi}\right)$, $0 \leqq \phi \leqq 2 \pi$, has at most $(n-1)^{2}$ vertices. Furthermore this bound is sharp, i.e. for any integer $n>1$ we can find a polynomial $p$ of degree $n$ such that $p\left(e^{i \phi}\right)$ has exactly $(n-1)^{2}$ vertices. let

Proof. As before let $g(t, x)$ be the Dieudonné polynomial for $p$ and

$$
g^{*}(t, x)=x^{n-1} \overline{g(\bar{t}, 1 / \bar{x})} .
$$

Each vertex of $p\left(e^{i \phi}\right)$ corresponds to at least two pairs $(t, x)$ and $(-t,-x)$ such that $g(t, x)=g^{*}(t, x)=0$ and $g(-t,-x)=g^{*}(-t,-x)=0$, that is, both $(t, x)$ and $(-t,-x)$ are intersection points of the algebraic curves given by $g(t, x)=0$ and $g^{*}(t, x)=0$. Now the degree of $g$ (in $t$ and $x$ jointly) is $2(n-1)$ and the degree of $g^{*}$ is $n-1$. If $g$ and $g^{*}$ are relatively prime, that is, if the curves have no common component, then by Bézout's theorem (Walker $[9$, p. 59]) the maximum number of intersection points is the product of the orders, $2(n-1)^{2}$. Thus the first part of the theorem is proved if we show that $g$ and $g^{*}$ are relatively prime.

To show that $g$ and $g^{*}$ are relatively prime as polynomials in $K[t, x]$ we show that the degree in $x$ of any common divisor must be zero, and that furthermore there is no common divisor in $K[t]$. If $g$ and $g^{*}$ have a common factor which is a function of $x$ then the resultant $R(t)$ is identically zero, but as we have seen above $R(t)$ is of degree $2(n-1)^{2}$. Thus we need only check that $g$ and $g^{*}$ have no common divisor $h(t)$. If $g$ is divisible by a function $h(t)$ then $h$ must divide $U_{n-1}$ and therefore must have a zero $t_{0}=$ $\cos (k \pi / n)$ for some integer $k, 1 \leqq k \leqq n-1$. Then

$$
g\left(t_{0}, x\right)=\left(p\left(x e^{i k \pi / n}\right)-p\left(x e^{-i k \pi / n}\right)\right) / x\left(e^{i k \pi / n}-e^{-i k \pi / n}\right)=0
$$

identically in $x$. Thus $p(x) \equiv p\left(x e^{2 k \pi i / n}\right)$ and we may write $p(x)=g\left(x^{l}\right)$ 
where $e^{2 k \pi i / n}$ is a primitive $l$ th root of unity and $g$ is a polynomial, and this contradicts our hypothesis.

To prove that the theorem is sharp, let $p(z)=\varepsilon z+z^{n}, \varepsilon>0$. We will show that for $\varepsilon$ small enough the curve $p\left(e^{i \phi}\right)$ has exactly $(n-1)^{2}$ vertices. We have

$$
g(t, x)=\varepsilon+U_{n-1}(t) x^{n-1} .
$$

If $\left|U_{n-1}(t)\right|=\varepsilon$, then $g(t, x)$ will have all of its zeros on $|x|=1$. Since $U_{n-1}(t)$ has simple zeros at $t=\cos (k \pi / n), k=1,2, \cdots, n-1$, it follows that for $\varepsilon$ small enough there are $2(n-1)$ values of $t$ in the interval $(-1,0) \cup$ $(0,1)$ for which $\left|U_{n-1}(t)\right|=\varepsilon$. Since $\left|U_{n-1}(t)\right|=\left|U_{n-1}(-t)\right|, n-1$ of these values are in the interval $0<t<1$. Let $t_{1}, t_{2}, \cdots, t_{n-1}$ be these values, where we assume that $t_{k}=t_{k}(\varepsilon), k=1, \cdots, n-1$, are continuous functions of $\varepsilon$ for $\varepsilon$ small. Now $g\left(t_{k}, x\right)$ has roots $\omega_{j k}, j=1, \cdots, n-1$, where the $\omega_{j k}, j=1, \cdots, n-1$, are the $n-1$ roots of 1 if $U_{n-1}\left(t_{k}\right)=\varepsilon$ and the $n-1$ roots of -1 if $U_{n-1}\left(t_{k}\right)=-\varepsilon$. We have

$$
p\left(\omega_{j k} e^{i \theta_{k}}\right)=p\left(\omega_{j k} e^{-i \theta_{k}}\right), \quad j, k=1, \cdots, n-1,
$$

where $\cos \theta_{k}=t_{k}, 0<\theta_{k}<\pi / 2$. We need only show that for $\varepsilon$ small enough the $(n-1)^{2}$ values $p\left(\omega_{j k} e^{i \theta_{k}}\right)$ are distinct. We can do this by showing that the values $\lim _{\varepsilon \rightarrow 0} p\left(\omega_{j k} e^{i \theta_{k}(\varepsilon)}\right)$ are distinct. We omit the details. This completes the proof of the theorem.

As a simple consequence of this theorem we note that a polynomial $p$ cannot map $|z|<1$ conformally onto a domain with a slit, for in this case $p\left(e^{i \phi}\right)$ would have an infinite number of vertices.

Univalent polynomials. We now show how the above discussion relates to the problem of computing polynomials univalent in $|z|<1$. We have found the maximum possible number of vertices for the curve $p\left(e^{i \phi}\right)$. At the opposite extreme, if $p\left(e^{i \phi}\right)$ has no vertices then it is a Jordan curve and $p(z)$ is univalent in $|z|<1$. In fact, let $p(z)=z+a_{2} z^{2}+\cdots+a_{n} z^{n}$. We let $V_{n}$ be the set of all points $\left(a_{2}, \cdots, a_{n}\right) \in K^{n-1}$ such that $p(z)$ is univalent in $|z|<1$.

THEOREM 2. $\left(a_{2}, \cdots, a_{n}\right) \in$ Int $V_{n}$ if and only if the curve $p\left(e^{i \phi}\right)$ has no vertices or cusps.

Proof. We first note that saying $p\left(e^{i \phi}\right)$ has no vertices or cusps is equivalent to saying that $g(t, x)$ has no zeros on $|x|=1$ for $-1 \leqq t \leqq 1$, where $g(t, x)$ is the Dieudonné polynomial associated with $p(z)$. Let $\delta$ be the minimum value of $|g(t, x)|$ for $|x|=1$ and $-1 \leqq t \leqq 1$. If $p\left(e^{i \phi}\right)$ has no vertices or cusps, then $\delta>0$. Now $\delta$ is a continuous function of the coefficients of $p$. Thus there is a neighborhood $N$ of $\left(a_{2}, \cdots, a_{n}\right)$ for which 
the corresponding polynomials map the unit circle onto a Jordan curve and are therefore univalent. Therefore $N \subset V_{n}$.

Conversely if $\left(a_{2}, \cdots, a_{n}\right) \in$ Int $V_{n}$, the polynomial $q(z)=(1 / r) p(r z)$, $r>1$, is univalent in $|z|<1$ for $r$ near 1 . Now $q\left(e^{i \phi} / r\right)=p\left(e^{i \phi}\right) / r$ and so $p\left(e^{i \phi}\right)$ can have no vertices. Likewise $p^{\prime}\left(e^{i \phi}\right) \neq 0$ for $0 \leqq \phi \leqq 2 \pi$. This completes the proof of Theorem 2.

As before let $R(t)$ be the resultant of $g(t, x)$ and $g^{*}(t, x)$. We prove the following:

THEOREM 3. $\left(a_{2}, \cdots, a_{n}\right) \in$ Int $V_{n}$ if and only if $R(t)$ has no zeros for $-1 \leqq t \leqq 1$.

Proof. We recall that if $t$ is real and if $x_{1}, \cdots, x_{n-1}$ are the zeros of $g(t, x)$, then $1 / \bar{x}_{1}, \cdots, 1 / \bar{x}_{n}$ are the zeros of $g^{*}(t, x)$. Now $R(t)$ is the resultant of these two polynomials so if $R(t)$ has no zeros for $-1 \leqq t \leqq 1$ then $g(t, x)$ has no zeros on $|x|=1$ for $-1 \leqq t \leqq 1$ and therefore, by Theorem $2,\left(a_{2}, \cdots, a_{n}\right) \in$ Int $V_{n}$. Conversely if $\left(a_{2}, \cdots, a_{n}\right) \in \operatorname{Int} V_{n}$ then $p\left(r e^{i \phi}\right)$ has no cusps or vertices for $r \leqq 1$ and therefore $g(t, x)$ has all of its zeros in $|x|>1$ for $-1 \leqq t \leqq 1$. Thus for $-1 \leqq t \leqq 1$, all the zeros of $g^{*}(t, x)$ are in $|x|<1$. Thus $g^{*}(t, x)$ and $g(t, x)$ have no common divisors and therefore $R(t) \neq 0$ for $-1 \leqq t \leqq 1$. This completes the proof of Theorem 3 .

As an example of Theorem 3, we compute the real cross-section of $V_{3}$. If $p(z)=z+a_{2} z^{2}+a_{3} t^{3}$ where $a_{2}$ and $a_{3}$ are real we have

$$
\begin{aligned}
g(t, x)= & 1+2 a_{2} t x+a_{3}\left(4 t^{2}-1\right) x^{2}, \\
g^{*}(t, x)= & a_{3}\left(4 t^{2}-1\right)+2 a_{2} t x+x^{2}, \\
R(t)= & \left(4 a_{3} t^{2}+2 a_{2} t+1-a_{3}\right)\left(4 a_{3} t^{3}-2 a_{2} t+1-a_{3}\right) \\
& \times\left(4 a_{3} t^{2}-\left(1-a_{3}\right)\right)^{2} .
\end{aligned}
$$

Now $R(t)$ has no zeros in $-1 \leqq t \leqq 1$ if and only if no factor of $R(t)$ has a zero for $-1 \leqq t \leqq 1$. An easy computation shows that this happens if and only if $\left(a_{2}, a_{3}\right) \in C_{1} \cap\left(C_{2} \cup C_{3}\right)$ where

$$
\begin{aligned}
& C_{1}=\left\{\left(a_{2}, a_{3}\right)|| a_{3} \mid<\frac{1}{3}\right\}, \\
& C_{2}=\left\{\left(a_{2}, a_{3}\right)|| 1+3 a_{3}|>| 2 a_{2} \mid\right\}, \\
& C_{3}=\left\{\left(a_{2}, a_{3}\right) \mid 4 a_{2}^{2}-16 a_{3}\left(1-a_{3}\right)<0\right\} .
\end{aligned}
$$

The closure of $C_{1} \cap\left(C_{2} \cup C_{3}\right)$ is the real cross-section of $V_{3}$. See Brannan [1].

In general it is not so easy to compute when $R(t)$ has no zeros for $-1 \leqq t \leqq 1$ since in general $R(t)$ will not factor as above. Theoretically the problem can be solved using Sturm sequences or Hermitian forms (see Uspensky [7]) but the computations are difficult. A computation of $V_{3}$ for 
arbitrary $a_{2}$ and $a_{3}$ is done by Brannan [1]. Computations on $V_{4}$ have been done by Michel [4] and Brannan [1]. In both of these references the method of Cohn [2] is used to determine when $g(t, x)$ has no zero in $|x| \leqq 1$. This method is equivalent to a method of Schur [5] using a Hermitian form. Our resultant $R(t)$ is the discriminant of this Hermitian form.

\section{REFERENCES}

1. D. A. Brannan, Coefficient regions for univalent polynomials of small degree, Mathematika 14 (1967), 165-169. MR 36 \#3971.

2. A. Cohn, Uber die Anzahl der Wurzeln einer linear algebraischen Gleichung in einem Kreise, Math. Z. 14 (1922), 110-148.

3. J. Dieudonné, Recherches sur quelques problèmes relatifs aux polynômes et aux fonctions bornées d'une variable complexe, Thèse, Ann. Sci. École Norm. Sup. (3) 48 (1931).

4. C. Michel, Eine Bemerkung zu schlichten Polynomen, Bull. Acad. Polon. Sci. Sér. Sci. Math. Astronom. Phys. 18 (1970), 513-519. MR 43 \#6423.

5. I. Schur, Über Potenzreiben, die in Innern des Einheitskreises beschränkt sind, J. Reine Angew. Math. 148 (1918), 122-145.

6. C. J. Titus, The combinatorial topology of analytic functions on the boundary of a disk, Acta Math. 106 (1961), 45-64. MR 29 \#3652.

7. J. V. Uspensky, Theory of equations, McGraw-Hill, New York, 1948.

8. B. L. van der Waerden, Modern algebra. Vol. 1, Springer, Berlin, 1930; English transl., Ungar, New York, 1949. MR 10, 587.

9. R. J. Walker, Algebraic curves, Princeton Math. Series, vol. 13, Princeton Univ. Press, Princeton, N.J., 1950. MR 11, 387.

Department of Mathematics, Florida State University, Tallahassee, Florida 32306 